\title{
CORRIGENDUM
}

\section{Understanding frailty: meanings and beliefs about screening and prevention across key stakeholder groups in Europe - CORRIGENDUM}

RACHEL L. SHAW, HOLLY GWYTHER, CAROL HOLLAND, MARIA BUJNOWSKA, DONATA KURPAS, ANTONIO CANO, MAURA MARCUCCI, SILVIA RIVA and BARBARA D'AVANZO

doi:10.1017/So144686X17000745, published online by Cambridge University Press 11 October 2017.

MARIA BUJNOWSKA-FEDAK's surname was incorrectly typeset.

\section{Reference}

Shaw, R. L., Gwyther, H., Holland, C., Bujnowska-Fedak, M., Kurpas, D., Cano, A., Marcucci, M., Riva, S. and D'Avanzo, B. Understanding frailty: meanings and beliefs about screening and prevention across key stakeholder groups in Europe. Ageing E Society, published by Cambridge University Press, 11 October 2017. doi: 10.1017/So144686X17000745 